\title{
Real time executable model for dynamic heat flow analysis of a solar hydrogen reactor
}

\author{
Echtzeitmodell zur dynamischen Wärmestromanalyse eines solarbetriebenen \\ Wasserstoffreaktors
}

https://doi.org/10.1515/teme-2019-0128

Received September 9, 2019; accepted March 29, 2020

Abstract: A real time executable model developed for dynamic heat analysis of a solar hydrogen reactor is described and characterized. To calculate the local distribution of radiation caused by multiple reflection inside the solar receiver the radiosity method is used. Significant optical characteristics including reflectance, transmittance and absorption are investigated related to the used materials and operating conditions. Furthermore, the influence on the model behavior is presented by variation of optical parameters. Simulation results are presented, which show good agreement with experimental data.

Keywords: Solar fuels, hydrogen, water splitting, $\mathrm{CO}_{2}$ reduction, concentrated solar power, renewable energy, thermodynamics.

Zusammenfassung: Ein in Echtzeit ausführbares Modell für die dynamische Wärmeanalyse eines solaren Wasserstoffreaktors wird beschrieben und charakterisiert. Zur Berechnung der lokalen Strahlungsverteilung durch Mehrfachreflexion im Reaktor wird die Nettostrahlungsmethode verwendet. In Bezug auf die verwendeten Materialien und Betriebsbedingungen werden signifikante optische Eigenschaften wie Reflexion, Transmission und Absorp-

\footnotetext{
*Corresponding author: Steffen Menz, Laboratory of Electrical Power Engineering, Rheinische Fachhochschule Köln (RFH), University of Applied Science, Cologne, Germany, e-mail: Steffen.Menz@rfh-koeln.de

Jörg Lampe, System theory and Mathematics, Engineering Faculty, Rheinische Fachhochschule Köln (RFH), University of Applied Sciences, Cologne, Germany

Uwe Tröltzsch, ITK engineering, Cologne, Germany Philipp Weiler, Mechanical Engineering, Rheinische Fachhochschule Köln (RFH), University of Applied Science, Cologne, Germany Arne Pahl, Fundamentals of Electrical Engineering, Helmut Schmidt University Hamburg, Hamburg, Germany

Thomas Fend, German Aerospace Center (DLR), Institute of Solar Research, Cologne, Germany

Thomas Seeger, University of Siegen, Engineering Thermodynamics, D-57076 Siegen, Germany; and Center for Sensor Systems (ZESS), University of Siegen, 57076 Siegen, Germany
}

tion untersucht. Das Modellverhalten wird durch die Variation optischer Parameter dargestellt. Abschließend werden Simulationsergebnisse vorgestellt, welche gut mit experimentellen Daten übereinstimmen.

Schlagwörter: Solare Brennstoffe, Wasserstoff, Wasserspaltung, $\mathrm{CO}_{2}$ Reduzierung, konzentrierte Solarstrahlung, erneuerbare Energie, Thermodynamik.

\section{Introduction}

Solar fuels offer promising potential to reduce $\mathrm{CO}_{2}$ emissions in the electricity, heat and transport sectors. This work is based on the hydrogen production process via a two-step thermochemical redox cycle by use of concentrated solar power (CSP) [1, 2].

Using ceria as reactive material, the method offers a theoretical solar-to-fuel efficiency up to $30 \%$ [3] that exceeds the efficiency of established photovoltaic combined with subsequent electrolysis. Currently, research is carried out in thermal analysis $[4,5,6]$, reactive materials $[7,8]$, reactor design $[9,10]$, plant operation $[11,12]$ and technoeconomic analysis [13, 14]. In order to increase efficiency, a fully automated plant operation has to be developed. Thus, a detailed simulation model is required for state estimation of several aspects including fluid flow, heat transfer and chemical reactions. The proposed model will be executed in real time using a programmable logic controller (PLC) controlling the whole hydrogen production process by means of system temperature and mass flow rates.

The solar receiver is one of the most important core components of the reactor, see Figure 1. Several design studies have improved the reactor geometry to ensure an efficient heat transfer by use of computationally expensive ray tracing methods and highly detailed pore level simulations $[15,16]$. The thermal performance has strong influence on the working conditions, since the thermochemical cycle is highly temperature dependent. The focus of this paper is on the model part of local temperature distribu- 


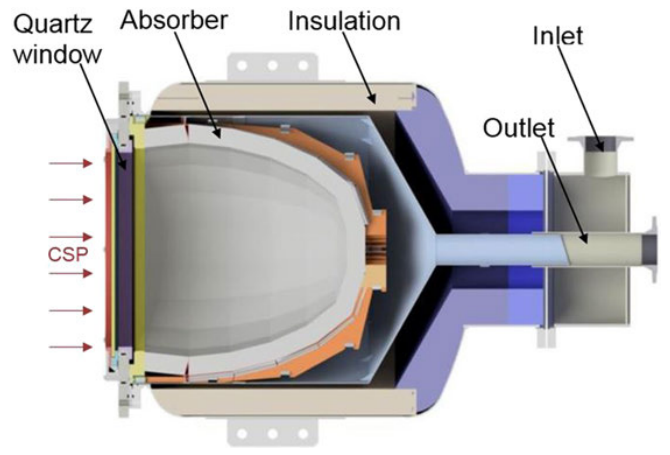

Figure 1: Thermochemical reactor including the main components absorber, quartz window, insulation, inlet and outlet.

tion throughout the volumetric absorber depending on solar and thermal radiation. Therefore, a simplified fast executable heat flow model considering several aspects of the incoming solar flux profile, the reactor geometry and material properties has been developed. To predict the reactors complete thermal behavior under different operating conditions in more detail, the presented heat flow model has to be coupled to additional domains of multiple dynamic varying gas mixtures and chemical processes.

\section{Plant operation}

The plant concept has been developed by the German Aerospace Center (DLR) within several projects [11, 15, 17]. For the current plant operation, the reactor is mounted on a solar tower and irradiated by a heliostat field. Solar power enters the reactor through the quartz window and heats up the volumetric metal oxide absorber. The absorber front side is made of porous ceria, used as catalytic material for hydrogen production via water splitting.

The used two-stage thermochemical redox cycle is illustrated in Figure 2, together with the associated chemical reactions of thermal reduction, see Equation (1); and oxidation, see Equation (2).

In the endothermic reduction step, outgoing from oxidized state $M O_{o x}$ in Figure 2 and formulated by the associated chemical Equation (1), oxygen is separated from the cerium oxide at a temperature level of $1400^{\circ} \mathrm{C}$. The separated oxygen molecules are carried away using nitrogen as back flushing gas. In the exothermic oxidation step, outgoing from reduced state $M O_{\text {red }}$ in Figure 2 and formulated by Equation (2), steam is decomposed at temperatures between 800 and $1100{ }^{\circ} \mathrm{C}$ into its individual components, hydrogen and oxygen. The cerium oxide is enriched by oxy-

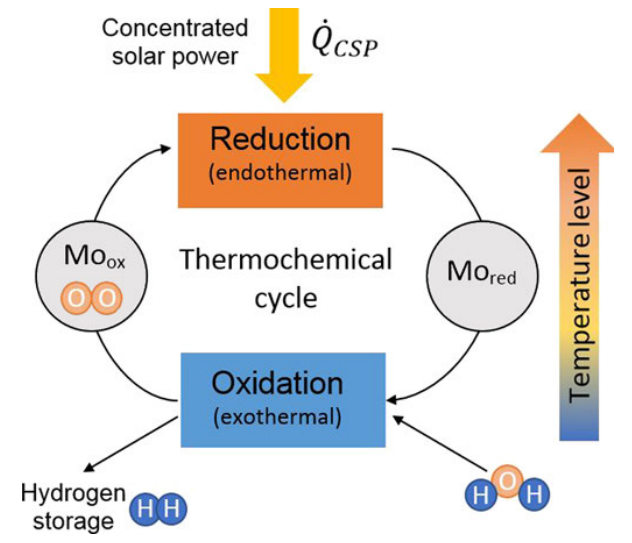

Figure 2: Thermochemical two-step metal redox cycle with the separated steps of thermal reduction and oxidation.

gen again; generated hydrogen leaves the reactor through the outlet. After the cerium oxide is saturated with oxygen, the process can be started again. In Equations (1) and (2), $\delta_{\text {ox }}$ and $\delta_{\text {red }}$ are the nonstoichiometric coefficients for reduction and oxidation and $\Delta h_{\text {red }}$ and $\Delta h_{\text {ox }}$ represents the change in enthalpy in $\mathrm{J} / \mathrm{mol}$ for the reduction and oxidation steps.

$$
\begin{aligned}
& \mathrm{CeO}_{2-\delta_{\text {ox }}} \stackrel{+\Delta h_{\text {red }}}{\longrightarrow} \mathrm{CeO}_{2-\delta_{\text {red }}}+\frac{\left(\delta_{\text {red }}-\delta_{\text {ox }}\right)}{2} \mathrm{O}_{2} \\
& \mathrm{CeO}_{2-\delta_{\text {red }}}+\left(\delta_{\text {red }}-\delta_{\text {ox }}\right) \mathrm{H}_{2} \mathrm{O} \stackrel{-\Delta h_{\mathrm{ox}}}{\longrightarrow} \mathrm{CeO}_{2-\delta_{\text {ox }}}+\left(\delta_{\text {red }}-\delta_{\text {ox }}\right) \mathrm{H}_{2}
\end{aligned}
$$

The two operating temperatures are crucial for the efficiency of the complete plant set up. In the reduction step, high temperatures offer several advantages of fast reaction rates following Arrhenius law and high extent of reduction efficiency by means of change in oxygen stoichiometry. According to [18], the extent of non-stoichiometry at equilibrium state is $\delta=0.0048$ at $1200{ }^{\circ} \mathrm{C}, \delta=0.0126$ at $1300^{\circ} \mathrm{C}$ and $\delta=0.033$ at $1400^{\circ} \mathrm{C}$ at $10^{-5}$ bar oxygen partial pressure. Practical limiting factors are material properties of the quartz window and sealing. In the exothermic oxidation step, the temperature has to be large enough such that enough activation energy is provided, but small enough for a low back-reaction rate. With regard to the overall efficiency, the temperature gap is an important aspect. The operating temperatures result in different cycle times for both steps caused by additional heating up and cooling down times. Due to the thermal management, three reactors are working simultaneously for a quasi-continuous hydrogen production. One reactor in the oxidation step and two reactors in the reduction step. With the use of suitable control strategies, the process temperatures can be optimized in future investigations. 


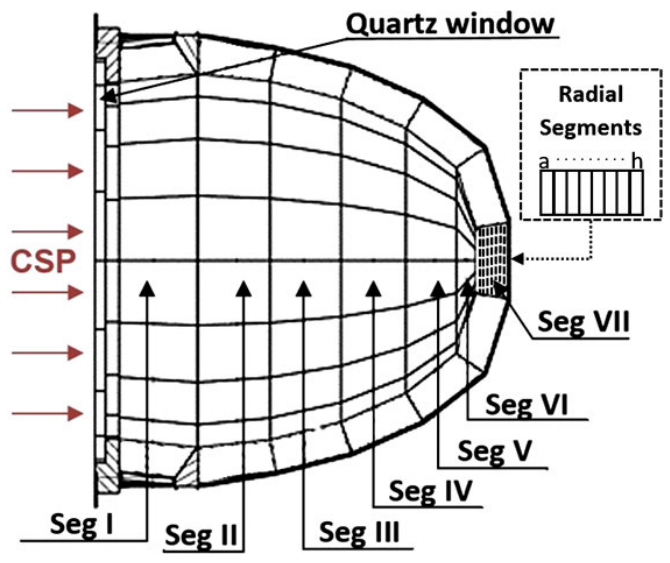

Figure 3: Cell segmentation of the solar absorber used for the simplified real time model including segments I-VII and radial sublayers a-h exemplary sketched in Seg. VII.

\section{Heat transfer model}

A complex heat transfer model of the solar reactor is created within Matlab's physical development environment Simscape by use of the versions 2017b-2019a [19]. Simscape includes various toolboxes with basic models for fluid flow and heat transfer systems, considering essential governing equations. It is possible to customize the basic models and to develop additional blocks with any desired functionality. To discretize the reactor geometry, the cell method is used. After a process analysis of gas flow and heat transfer behavior inside the reactor, several sets of segment classification in different abstraction levels have been defined.

Figure 3 shows a schematic of the used cell classification of the illustrated simplified real time model version. The absorber is discretized by six annular segments (Seg. I-VI) due to axis symmetry and one circular segment (Seg. VII). Each segment is subdivided into eight radial sublayers $\mathrm{a}-\mathrm{h}$, in order to additionally calculate a radial temperature gradient inside the porous absorber. The radial segments a-d have a length of $5 \mathrm{~mm}$, while the last four segments $\mathrm{e}-\mathrm{h}$ have a radial length of $10 \mathrm{~mm}$. The first sublayer (a) represents the surface, which additionally considers the spatial heat transfer caused by solar and thermal radiation. The quartz window is implemented as one single segment. The temperature is calculated in each segment, which is assumed to be isothermal.

By the use of intended mesh refinement and convergence analysis, optimal cell arrangements can be determined to establish rapid executable approximation as well as computational intensive high accurate model versions in ongoing work.

\section{Governing equations}

In the following, a set of governing equations according to fundamental model components is illustrated. Generally, the gas domain is following the ideal gas law

$$
p \cdot V=m \cdot R_{\mathrm{S}} \cdot T
$$

where $p$ and $T$ are the pressure and temperature of the gas volume in bar and Kelvin, $V$ is the Volume in $\mathrm{m}^{3}, m$ is the mass in $\mathrm{kg}$ and $R_{S}$ is the specific gas constant in $\mathrm{J} /(\mathrm{kg} \cdot \mathrm{K})$. Mass conservation is related to mass flow rates and the dynamics of the pressure and temperature of gas volume.

$$
\frac{\partial M}{\partial p, c} \cdot \frac{\partial p}{\partial t}+\frac{\partial M}{\partial T, c} \cdot \frac{\partial T}{\partial t}=\dot{m}_{\mathrm{in}}+\dot{m}_{\text {out }}
$$

The term $\partial M / \partial p, c$ is the partial derivative of the gas mass of a discrete volume element with respect to pressure at constant temperature and volume and $\partial M / \partial T, c$ is the partial derivative of the gas mass with respect to temperature at constant pressure and volume. Further, $t$ is the time in seconds, $\dot{m}_{\text {in }}$ and $\dot{m}_{\text {out }}$ are the mass flow rates at the segment inlet and outlet in $\mathrm{kg} / \mathrm{s}$, whereby inflowing gas is related to positive sign and effluent gas to a negative sign. Energy conservation relates the energy and heat flow rates to the dynamics of the gas volumes pressure and temperature of the gas volume

$$
\frac{\partial U}{\partial p, c} \cdot \frac{\partial p}{\partial t}+\frac{\partial U}{\partial T, c} \cdot \frac{\partial T}{\partial t}=\phi_{\text {in }}+\phi_{\text {out }}+\phi_{\text {add }}
$$

where $\partial U / \partial p, c$ is the partial derivative of internal energy of the gas volume with respect to pressure at constant temperature and volume and $\partial U / \partial T, c$ is the partial derivative with respect to temperature at constant pressure and volume. The variables $\phi_{\text {in }}, \phi_{\text {out }}$ and $\phi_{\text {add }}$ represent the energy flow rates in $\mathrm{W}$ at the inlet, outlet and additional energy flow by means of solar power or heat transfer from of adjacent segments. The momentum balance models the pressure drop due to momentum flux and viscous friction

$$
p_{\text {in }}-p_{\text {out }}=\left(\frac{\dot{m}_{\text {in }}+\dot{m}_{\text {out }}}{A}\right)^{2} \cdot\left(\frac{1}{\Delta \rho}\right)+\Delta_{p, f r}
$$

where $p_{\text {in }}$ and $p_{\text {out }}$ are the pressure at the inlet and the outlet of one element, $A$ is the area in $\mathrm{m}^{2}, \Delta \rho$ is the difference of density at the inlet and outlet and $\Delta_{p, f r}$ is additional pressure loss due to viscous friction. Further, the energy balance of thermal masses $\dot{Q}_{m}$ is defined as:

$$
\dot{Q}_{m}=c_{p} \cdot m \cdot \frac{d T}{d t}
$$

where $\dot{Q}$ is the heat flow in W and $c_{p}$ is the specific heat capacity of the material in $\mathrm{J} /(\mathrm{kg} \cdot \mathrm{K})$. Conductive heat transfer $\dot{Q}_{\text {cond }}$ between two material layers is governed by the 
Fourier law

$$
\dot{Q}_{\text {cond }}=k \cdot \frac{A}{d} \cdot\left(T_{1}-T_{2}\right)
$$

where $k$ is the thermal conductivity in $\mathrm{W} /(\mathrm{m} \cdot \mathrm{K})$ and $d$ is the distance, respective the material thickness in $\mathrm{m}$. Convective heat transfer $\dot{Q}_{\text {conv }}$ by means of fluid motion is governed by the Newton law of cooling

$$
\dot{Q}_{\mathrm{conv}}=\alpha \cdot A \cdot\left(T_{1}-T_{2}\right)
$$

where $\alpha$ is the convective heat transfer coefficient in $\mathrm{W} /\left(\mathrm{m}^{2} \cdot \mathrm{K}\right)$. Radiative heat transfer $\dot{Q}_{\mathrm{rad}}$ is given by:

$$
\dot{Q}_{\text {rad }}=h_{r} \cdot A \cdot\left(T_{1}^{4}-T_{2}^{4}\right)
$$

where $h_{r}$ is the radiation coefficient in $\mathrm{W} /\left(\mathrm{m}^{2} \cdot \mathrm{K}^{4}\right)$.

\subsection{Model components}

Various material properties, geometric and optical effects have strong influence on the spatial temperature distribution inside the solar receiver. In this subsection, the main aspects including solar flux, geometrical aspects, as well as optical and material properties of the used component models are described.

\section{Incoming solar flux profile}

The incoming solar flux profile depends on the heliostat field configuration. Important aspects are the position and alignment of active heliostats, as well as the distance from the heliostat field to the solar tower. Usually, the incoming solar flux profile is provided inhomogeneously which can lead to spatial irregularities of the temperature profile. Figure 4 shows the spatial temperature distribution on the absorber measured at the sun simulator laboratory Synlight [20] during the reduction step by use of a thermal camera. Due to the focus setting of the high-performance radiators, two hot spots are created on the absorber surface. To include the aspect of varying flux distribution in the model, the amount of solar flux provided on one segment $\dot{Q}_{\text {seg }}$ is calculated considering the total amount of incoming solar power $\dot{Q}_{\mathrm{CSP}, \mathrm{s}}$ in $\mathrm{W}$, the area as a relation of one single segment $A_{\text {seg }}$ and the total absorber front side area $A_{A}$ and the factor $f_{\text {flux }}$ to describe the local solar flux concentration which can be calculated by the number, position and angle of active spotlight modules, see Equation (11). Therewith, heliostat fields of varying size, settings and inhomogeneous intensities can be considered in a reasonable way.

$$
\dot{Q}_{\text {seg }}=\dot{Q}_{\mathrm{CSP}, \mathrm{s}} \cdot \frac{A_{\text {seg }}}{A_{A}} \cdot f_{\text {flux }}
$$

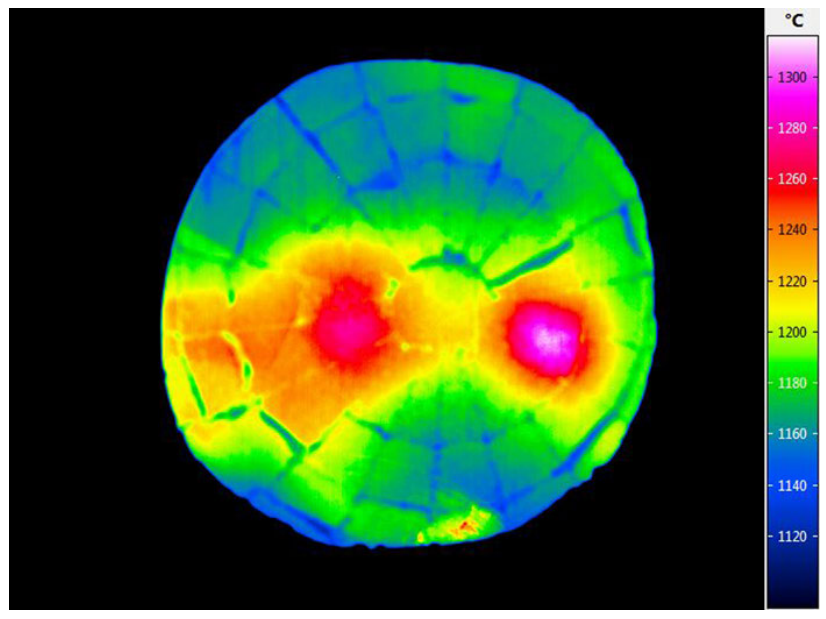

Figure 4: Absorber temperature profile taken during Synlight measurement campaign by thermal camera.

\section{Quartz window}

To evaluate the optical behavior of the quartz window and to determine the optical efficiency, material properties and plant design is considered.

The incoming radiation of the solar field is partly absorbed, transmitted and reflected by the window. In the current application, the incidence angle of incoming solar radiation is less than $20^{\circ}$, thus polarization can be neglected. The overall transmittance $\tau$, absorbance $\alpha$ and reflectance $r$ are initially calculated depending on the characteristics of the incoming radiation.

The optical factors of the quartz window for solar radiation are determined following Planck's law, where the sun represents a black body with the spectral specific radiation $M_{\lambda, s}$ and a temperature of $5777 \mathrm{~K}$. Due to Planck's law the required overall optical factors can be calculated for all temperatures with the help of $M_{\lambda, s}$ and the spectral factors $\tau \lambda, \alpha \lambda$ and $r \lambda$, which are available for the used window with steps of $1 \mathrm{~nm}$ from $250 \mathrm{~nm}$ to $5000 \mathrm{~nm}$ [21]. Figure 5 shows these spectral factors as well as the spectral specific radiation of the sun at $5777 \mathrm{~K}$. The overall optical factors are determined as follows [11]:

$$
i=\int_{250 \mathrm{~nm}}^{5000 \mathrm{~nm}} i_{\lambda} \cdot M_{\lambda, s}(T) \mathrm{d} \lambda, \quad i=\tau, \alpha, r
$$

For the used quartz window, an overall transmittance of $\tau=0.92$ is calculated while the absorbance and reflectance are determined to $\alpha=0.01$ and $r=0.07$, which represents a high efficiency for solar irradiation. The optical efficiency $\eta$ of the quartz window is assessed as the ratio between the incoming solar radiation and the amount 


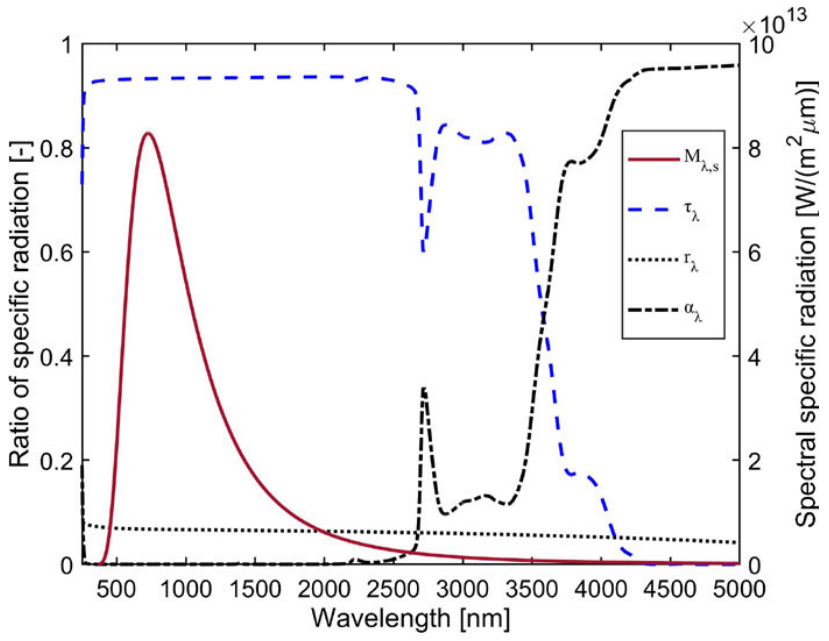

Figure 5: Spectral properties of transmission, absorption and reflection of the used quartz window together with spectral radiation of the sun as a black body of $5777 \mathrm{~K}$.

of radiation that is transmitted or absorbed by the window.

$$
\eta=(1-r)=0.93
$$

\section{Absorber}

The thermal performance of the reactor strongly depends on the geometrical design and absorber material properties. Design studies have revealed that a hemispherical absorber shape reduces thermal radiation losses back to the environment, in comparison to flat or conical shapes [15]. The used hemispherical absorber consists of 109 separate elements. A center disc and 6 annular segments each of which are made of truncated pyramid shaped ceria coated zirconia blocks of different size. The blocks are separated by a metal oxide fiber-mesh based insulation material. Typically, these kinds of materials have thermal conductivities of $0.4-2.7 \mathrm{~W} /(\mathrm{m} \cdot \mathrm{K})$ depending on their porosity. The absorber construction is illustrated schematically in Figure 3.

Several optical and thermodynamic material properties of ceria with comparable structure were investigated in $[4,16]$. The absorbance and reflectance of the material change as a function of temperature caused by a change in the material color. The temperature from start up to reduction phase ranges from $20^{\circ} \mathrm{C}$ to $1400^{\circ} \mathrm{C}$. According to [4], that leads to a change in reflection coefficient of about $17 \%$, i.e. in absolute values the reflection coefficient varies between $0.23-0.27$. The properties of porous materials strongly depends on grid arrangement and material structure. Relevant parameters of porosity $(\varepsilon=0.65)$, mean pore diameter $\left(d_{p}=2.27 \mathrm{~mm}\right)$ and cellularity (10.4 ppi) were measured by use of small samples at different locations and summarized as mean values, which are needed to apply volume averaged models. Corresponding parameters of specific surface area $\left(A_{0}=981 \mathrm{~m}^{-1}\right)$ and extinction coefficient $\left(\beta=500 \mathrm{~m}^{-1}\right)$ are determined according to [16]. Required material properties of effective thermal conductivity and effective heat capacity are measured by use of hot disc method [22] and adjusted to the course of high temperatures according to [4].

Inside the porous absorber, the temperature of solid and fluid phase may be different due to fluid flow and material properties. The Darcy-Forchheimer equation is used to account flow through porous media with $\mu_{\text {dyn }}$ as dynamic viscosity in Pa.s, $u$ as fluid velocity in $\mathrm{m} / \mathrm{s}, \rho_{f}$ as the fluid density in $\mathrm{kg} / \mathrm{m}^{3}, k_{\text {lam }}$ is the permeability for laminar flow in $\mathrm{m}^{2}$ and $k_{\text {turb }}$ is the permeability for turbulent flow in $\mathrm{m}[5]$.

$$
\nabla p=\frac{\mu_{\mathrm{dyn}}}{k_{\mathrm{lam}}} \cdot u+\frac{\rho_{f}}{k_{\mathrm{turb}}} \cdot|u| \cdot u
$$

Local thermal non-equilibrium models are widely applied to calculate the solid and fluid temperatures of porous media $[5,16]$. Two separate energy conservation equations for the fluid phase $(f)$ in Eq. (15) and solid phase $(s)$ in Eq. (16) are solved for a single representative volume element with $\varepsilon$ as the porosity, $c_{p f}$ and $c_{p s}$ as the specific heat capacity of fluid and solid in $\mathrm{J} /(\mathrm{kg} \cdot \mathrm{K}), T_{f}$ and $T_{s}$ as the temperature of fluid and solid in $\mathrm{K}$ and $k_{f}$ and $k_{s}$ as the thermal conductivity of fluid and solid in $\mathrm{W} /(\mathrm{m} \cdot \mathrm{K})$.

$$
\begin{aligned}
& \varepsilon \cdot \rho_{f} \cdot c_{p f} \frac{\partial T_{f}}{\partial t}+\left(\varepsilon \cdot \rho_{f} \cdot c_{p f}\right) \cdot u \cdot \nabla T_{f}=k_{f} \nabla \cdot \nabla T_{f}+Q_{f s} \\
& (1-\varepsilon) \rho_{s} \cdot c_{p s} \cdot \frac{\partial T_{s}}{\partial t}=k_{s} \nabla \cdot \nabla T_{s}-Q_{f s}
\end{aligned}
$$

Both equations are coupled by interfacial convective heat transfer $Q_{f s}$ calculated by use of the specific surface area $A_{0}$ and the corresponding heat transfer coefficient $\alpha_{f s}$ calculated by use of the Nusselt-Reynolds correlation [16].

$$
Q_{f s}=\alpha_{f s} \cdot A_{0} \cdot\left(T_{s}-T_{f}\right)
$$

The diffusion of radiative flux $q_{r}$ in $\mathrm{W} / \mathrm{m}^{2}$ is given by the Rosseland equation with $\sigma$ as Stefan-Boltzmann constant in $\mathrm{W} /\left(\mathrm{m}^{2} \cdot \mathrm{K}^{4}\right)$ and $\omega$ as index of refraction [5].

$$
q_{r}=-\frac{16 \cdot \sigma \cdot \omega^{2} \cdot T_{s}^{3}}{3 \cdot \beta} \cdot \nabla T_{s}
$$

Additional heat flow due to chemical reaction $\dot{Q}_{\text {reaction }}$ is calculated according to [23] with respect to the amount of ceria $n_{\text {mol }}$ in mol.

$$
\dot{Q}_{\text {reaction }}=\frac{d \delta}{d t} \cdot \Delta h \cdot n_{\mathrm{mol}}
$$


The change in oxygen content of the corresponding absorber element depends on the reaction rate $d \delta / d t$ in $\mathrm{s}^{-1}$, which is calculated according to [23] with $\delta$ as the current oxygen stoichiometry acc. to Equations (1) and (2), $x$ as the theoretical maximum change in stoichiometry, $A_{\text {red }}$ as the frequency factor for reduction in $\mathrm{s}^{-1}$ and $A_{\mathrm{ox}}$ the frequency factor for oxidation in $s^{-1} \cdot \mathrm{bar}^{-n}, E_{a, \text { red }}$ and $E_{a, 0 x}$ are the activation energies for reduction and oxidation in $\mathrm{kJ} / \mathrm{mol}, P_{o 2}$ is the partial oxygen pressure in bar and $n$ is the oxygen gas power dependency:

$$
\frac{d \delta}{d t}=(x-\delta) A_{\mathrm{red}} \cdot e^{\frac{-E_{a, \text { red }}}{R \cdot T}}-\delta \cdot P_{o 2}^{n} \cdot A_{\mathrm{ox}} \cdot e^{\frac{-E_{a, 0 x}}{R \cdot T}}
$$

The change in reaction enthalpy $\Delta h$ in $\mathrm{J} / \mathrm{mol}$ is calculated according to [24], whereby reduction is related to a positive sign and oxidation to a negative sign.

$\Delta h(\delta)=\left(478-1158 \cdot \delta+1790 \cdot \delta^{2}+23368 \cdot \delta^{3}-6492 \cdot \delta^{4}\right) \cdot 10^{3}$

\subsection{Radiosity method}

To model the spatial thermal distribution caused by multiple reflections between the participating reactor parts the radiosity method is applied, which presents a numerical solution of the shading equation of the finite element method [25]. The segmentation of the absorber surface into seven elements as shown in Figure 3 is used, where each segment represents a small element surface with an approximated constant intensity distribution. For this method the surface reflection is assumed to be diffuse and thus generates the same intensity in all directions. The approximated radiative heat exchange $\dot{Q}$ in $\mathrm{W}$ for each segment is given by the following two Equations (22) and (23), which consider the radiosity $H_{i}$ of the surface $i$.

$$
\begin{aligned}
& \dot{Q}_{i}=\frac{A_{i} \epsilon_{i}}{\left(1-\epsilon_{i}\right)}\left(\sigma T_{i}^{4}-H_{i}\right) \\
& \dot{Q}_{i}=A_{i} \sum_{j=1}^{n} F_{i j}\left(H_{i}-H_{j}\right)
\end{aligned}
$$

Equation (22) describes the heat transfer balance for the surface element $i$ as the amount of emitted radiation $M_{i}$ and reflected irradiation $r_{i} E_{i}$ using the radiosity $H$ of the surface in $\mathrm{W} / \mathrm{m}^{2}$, see Figure 6 . Furthermore, $\epsilon$ is the emissivity, $T$ is the temperature in $\mathrm{K}$ and $A$ the area in $\mathrm{m}^{2}$. Equation (23) contains the radiosity $H_{j}$ of surrounding surfaces including geometrical view factors $F$, which consider the geometrical position of the surfaces $i$ and $j$ towards each other. The localized heat distribution is determined

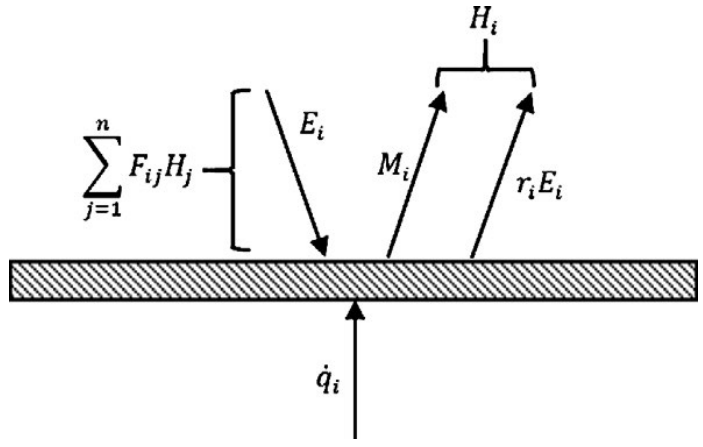

Figure 6: Illustration of energy balance and radiosity method as example for surface element $i$.

by a numerical solution of a resulting system of equations. Analogously, two separate physical networks for thermal and solar radiation are developed to calculate a heat profile of the absorber depending on the heat flux density $\dot{q}_{i}$ in $\mathrm{W} / \mathrm{m}^{2}$, based on a schematic shown in Figure 6 .

Note that the quartz window can be described as a semitransparent body. To ensure a correct application of the radiosity method for this component the above system of equations is extended by adding the transmissive characteristics of the used quartz glass [26].

\subsection{Model overview}

The heat transfer model has been developed in the subdivided parts of solar radiation and thermal radiation. In Figure 7, an overview of the considered quantities and effects is illustrated. At the quartz window, incoming concentrated solar radiation $\left(\dot{Q}_{\mathrm{CSP}, s}\right)$ is partly reflected $\left(\dot{Q}_{\mathrm{CSP}, \mathrm{s}, W, r}\right)$, absorbed $\left(\dot{Q}_{\mathrm{CSP}, s, W, a}\right)$ and transmitted $\left(\dot{Q}_{\mathrm{CSP}, s, W, t}\right)$. Due to absorption, energy is taken up by the quartz window which leads to a change in material temperature $\left(T_{W}\right)$. The transmitted solar radiation enters the reactor and hits the absorber where it is partly absorbed $\left(\dot{Q}_{\mathrm{CSP}, s, A, a}\right)$. The absorption of thermal radiation $\left(\dot{Q}_{A, \mathrm{rad}, A, a}\right)$ coming from other segments $\left(\dot{Q}_{A, \text { rad }}\right)$ is considered analogously. Depending on the absorber material temperature $\left(T_{A}\right)$, the surface emits radiation. Solar and thermal reflection $\left(\dot{Q}_{\mathrm{CSP}, \mathrm{s}, A, r}, \dot{Q}_{A, \mathrm{rad}, A, r}\right)$ are provided to the other absorber segments and to the quartz window. The part of irradiation going to the quartz window is again partly absorbed $\left(\dot{Q}_{\mathrm{CSP}, s, W, a}, \dot{Q}_{A, \mathrm{rad}, W, a}\right)$ transmitted $\left(\dot{Q}_{\mathrm{CSP}, s, W, t}, \dot{Q}_{A, \mathrm{rad}, W, t}\right)$ or reflected back into the cavity $\left(\dot{Q}_{\mathrm{CSP}, \mathrm{s}, W, r}, \dot{Q}_{A, \mathrm{rad}, W, r}\right)$.

The gas is assumed ideal and not participating in the process of radiation heat exchange in the presented model version. Considered gas properties are specific enthalpy, specific heat, dynamic viscosity, and thermal conductivity. 


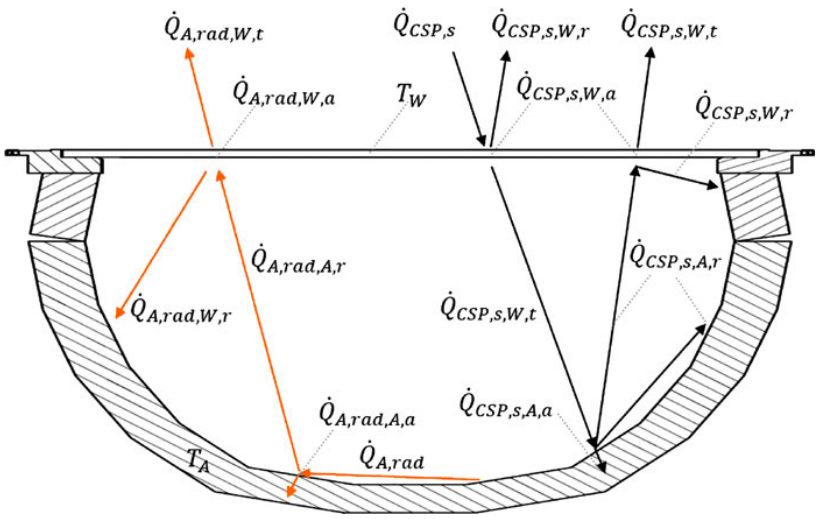

Figure 7: Heat transfer model scheme including the main model aspects of solar and thermal radiation.

Heat losses from the window to the environment $\dot{Q}_{\text {loss,qw }}$ and through the lateral surface $\dot{Q}_{\text {losssur }}$ are included by means of thermal radiation, conduction through the window and insulation and natural convection. The material temperature of the entire reactor is initially set to $25^{\circ} \mathrm{C}$; the ambient temperature is set to constant $30^{\circ} \mathrm{C}$. Nitrogen enters the reactor with an initial temperature of $25^{\circ} \mathrm{C}$, steam is fed by a vaporizer and entering the reactor with a temperature of $100^{\circ} \mathrm{C}$. Heat of incoming fluid through the inlet is defined as $\dot{Q}_{\text {fluid,in }}$ and $\dot{Q}_{\text {fluid,out }}$ leaves through the outlet. The energy balancing is defined as follows.

$$
\begin{aligned}
\dot{Q}_{\mathrm{CSP}, s}= & \dot{Q}_{A, \mathrm{rad}, A, a}+\dot{Q}_{\mathrm{CSP}, \mathrm{s}, A, a}+\dot{Q}_{\mathrm{CSP}, \mathrm{s}, W, a}+\dot{Q}_{A, \mathrm{rad}, W, a} \\
& +\dot{Q}_{A, \mathrm{rad}, W, t}+\dot{Q}_{\mathrm{CSP}, W, r}+\dot{Q}_{\text {loss }, q w}+\dot{Q}_{\text {loss }, \mathrm{sur}} \\
& -\dot{Q}_{\text {fluid,in }}+\dot{Q}_{\text {fluid,out }}+\dot{Q}_{\text {reaction }}
\end{aligned}
$$

Note that $\dot{Q}_{\text {reaction }}$ has not been considered in the simulation within this paper and thus set to zero.

\section{Results and discussion}

In this section, the thermodynamic model behavior is investigated. First, the local temperature distribution is compared to measurement results to validate the dynamic model behavior. Further, the model response by varying a selection of model parameters is discussed. The used measurement campaigns for validation were carried out in the high-flux solar simulator Synlight, which consists of 148 spotlight modules with an average radiation power of $2.7 \mathrm{~kW}_{\text {rad }}$ and a total facility power of approximately $400 \mathrm{~kW}_{\text {rad }}$. Due to the modularity, various flux distributions can be set up according to experimental requirements by means of different power levels and spotlight positioning. The spotlight modules are equipped with xenon
Table 1: Comparison of measured and simulated mean and maximum temperatures of each segment in $\mathrm{K}$.

\begin{tabular}{rrrrr}
\hline Segment & $\boldsymbol{T}_{\text {meas,mean }}$ & $\boldsymbol{T}_{\text {meas,max }}$ & $\boldsymbol{T}_{\text {sim,mean }}$ & $\boldsymbol{T}_{\text {sim,max }}$ \\
\hline I & 674 & 920 & 692 & 1015 \\
II & 731 & 1036 & 706 & 1028 \\
III & 693 & 1017 & 697 & 1010 \\
IV & 707 & 1003 & 697 & 1010 \\
V & 701 & 992 & 695 & 1004 \\
VI & 730 & 1024 & 703 & 1028 \\
VII & 770 & 1084 & 780 & 1142 \\
\hline
\end{tabular}

short arc lamps, the radiation spectrum similar to the solar spectrum. The provided heat flux and temperature can be measured by a thermal camera system and a radiometer [20]. During the campaigns, the general reactor functionality has been ensured by several heating and cooling cycles with large temperature ranges about more than five hours. The absorber temperature was measured by 55 thermocouples (Type K, Class 2; Average tolerance: $0.0075 \cdot T$; Sample rate: one second) embedded from the backside of every second element and one in the center disc.

\section{Dynamic model behavior}

To investigate the thermal model behavior, the simulation results of the local heat distribution are compared to measured temperature data. Therefore, the incoming solar radiation for generating the absorber temperatures is calculated according to the number of active spotlights during the experiment. Generally, at the segments I to VII the resulting temperature distribution is very similar. Table 1 shows an overview of the measured mean temperatures of the radial layer (h) of segments I-VII during the experiment in the second column and the corresponding simulated mean temperatures in the fourth column. The measured mean temperatures are ranged between 674$770 \mathrm{~K}$ (Seg. I and Seg. VII). Thus, the mean temperatures lie within in a $100 \mathrm{~K}$ range, which is also the case for the simulated data. In segment VII, the temperature is highest due to the central focus of the spotlight modules. In segment I, the temperature is lowest due to limited radiation and an indirect connection to the reactor front flange, which causes additional heat losses. In the third column the maximal measured temperature of segments I-VII are displayed, which corresponds to the simulated maximum values in the last column. The maximum measured shortterm temperature difference between two segments has been noticed with $272 \mathrm{~K}$ between segments I and III during the first heating-up phase at a simulation time of 1750 seconds. 


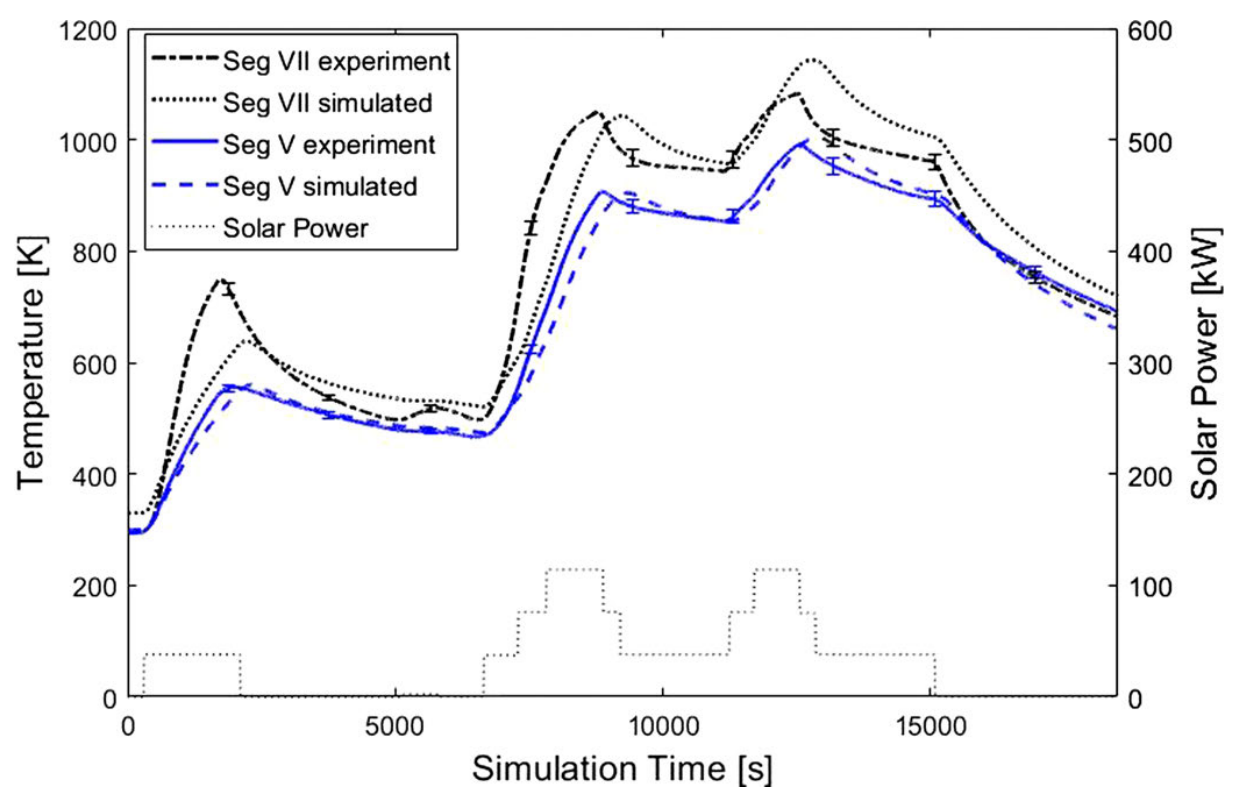

Figure 8: Measured absorber temperature and simulated temperature of segment $\mathrm{V}$ and VII depending on solar power.

Figure 8 shows a comparison of simulated and experimental data at a given concentrated solar input power as an example of segments V(h) and VII(h). The dotted curve displays the used solar power, calculated based on the number of active lamps, which shows that a large temperature range and several heating and cooling cycles are applied. The dashed and the solid line in Figure 8 correspond to the measured and simulated temperature in Seg. $\mathrm{V}(\mathrm{h})$, which show a very good agreement with a maximum difference of $67.2 \mathrm{~K}$ and a mean relative difference of $2.46 \%$. At the center disc, Seg. VII(h), higher differences with a maximum of $181.81 \mathrm{~K}$ and a relative difference of $6.98 \%$ can be detected. Because of the small surface area and small mass of segment VII, marginal inaccuracies of optical parameters can strongly affect the temperature calculation. Measurement tolerances are displayed by using error bars with a range of $\pm 3 \sigma_{s}$, with $\sigma_{s}$ as standard deviation. Due to the porous absorber structure, view factors can be subject to tolerances because of inhomogeneous scattering that may lead to local differences. As a result, optical view factors were adjusted by a parameter determination routine. An overview including the temperature differences is illustrated in Table 2.

\section{Influence of the absorber material reflection}

The reflection of the absorber layer strongly depends on the used material. One important aspect of the used ceria is the change in color at different temperatures. Other suitable redox materials like nickel ferrite provide different optical properties with higher absorption capacity [11].
Table 2: Maximum absolute difference $\Delta_{\text {max,abs }}$ in $\mathrm{K}$, absolute average difference $\Delta_{\mathrm{av} \text {,abs }}$ in $\mathrm{K}$ and average relative difference $\Delta_{\mathrm{av}, \text { rel }}$ in $\%$ of absorber segments I-VII as mean values of all temperature sensors per segment.

\begin{tabular}{rrrr}
\hline Segment & $\boldsymbol{\Delta}_{\text {max,abs }}$ in K & $\boldsymbol{\Delta}_{\text {av,abs }}$ in K & $\boldsymbol{\Delta}_{\text {av,rel }}$ in \% \\
\hline I & 103.95 & 31.20 & 5.46 \\
II & 119.23 & 29.68 & 4.32 \\
III & 55.33 & 21.03 & 3.54 \\
IV & 67.72 & 17.53 & 2.47 \\
V & 67.20 & 17.39 & 2.46 \\
VI & 146.50 & 31.77 & 4.51 \\
VII & 181.81 & 53.61 & 6.98 \\
\hline
\end{tabular}

To investigate the local temperature distribution depending on optical material properties in terms of difference between nickel ferrite and ceria, the reflection coefficient of the absorber material is varied from 0.1 to 0.4 . Thus, the absorption coefficient is adopted correspondingly from 0.9 to 0.6 , since the sum of reflection and absorption coefficient equals one. In Figure 9, the influence on the temperature of different reflection and absorption coefficients is illustrated by segments V, VII and the quartz window.

As expected, an increasing absorption coefficient and decreasing reflection coefficient leads to a higher absorber temperature. Less solar radiation is reflected to the quartz window and transmitted to the environment. By decreasing solar reflection and increasing absorber temperature, also thermal radiation from the absorber to the window in- 


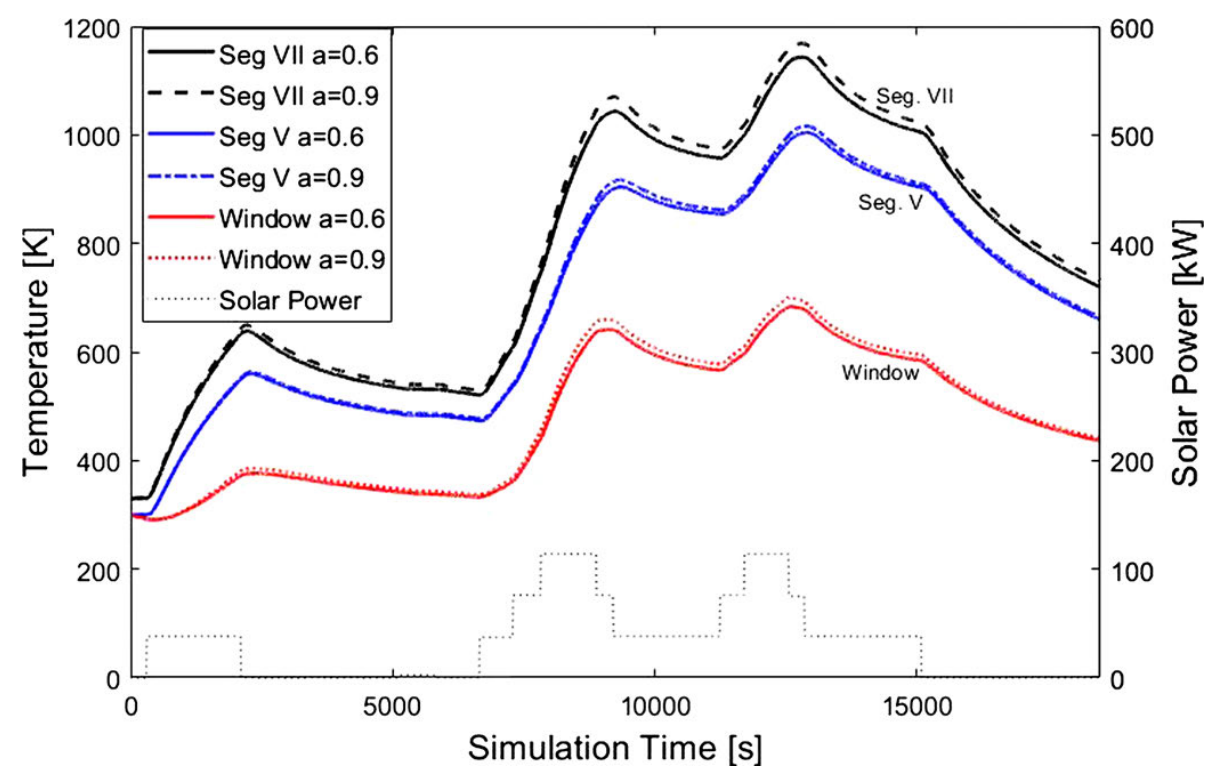

Figure 9: Absorber segment V, VII and window temperature depending on reflection and absorption coefficients.

creases, which effects a higher temperature of the quartz window. Reflected solar radiation from the absorber to the window has no big influence on the window temperature, since this part is almost completely transmitted. Maximum temperature differences of $13.5 \mathrm{~K}$ in Seg. V, $27.5 \mathrm{~K}$ in Seg. VII and $22.1 \mathrm{~K}$ in the quartz window are achieved. By use of volumetric absorbers, a large part of the radiation remains inside the cavity and only a small part reaches the outside through the quartz window. Depending on the absorber geometry, the effective absorption and reflection properties may change not significantly, which lead to small temperature differences [27].

\section{Influence of solar flux profile}

The local intensity of the solar flux profile depends on the configuration of the heliostat field. The influence on the local temperature distribution is illustrated by two different scenarios. In scenario 1, the thermal performance is investigated in case of a small solar field. Therefore, the flux is assumed to be provided nearly homogenously throughout the entire absorber surface. In scenario 2, the influence of large heliostat fields with a larger angle of incidence is investigated. Several hot spots in the middle region of the absorber surface are created, comparable to the temperature profile illustrated in Figure 4. The static local temperature distribution after $2 \mathrm{~h}$ radiation with constant $90 \mathrm{~kW}$ is shown in Figure 10 as an axisymmetric horizontal cross section through the absorber center. It can be seen that the local component of incoming solar flux strongly influences

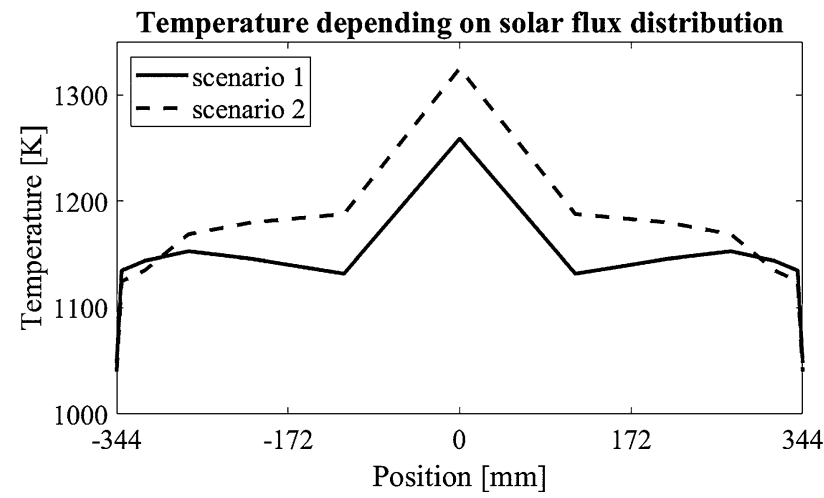

Figure 10: Absorber temperatures depending on the local incoming solar flux concentration.

the temperature distribution throughout the solar receiver. In comparison, radiation with several hotspots in the segments V-VII leads to higher temperatures in the middle region of the absorber. At the outer parts, the temperature decreases in comparison to the scenario of nearly homogeneous provided flux.

\section{Runtime analysis}

The simulations of the measurement campaign illustrated in Figure 8 requires a simulation runtime of $138 \mathrm{~s}$ for the measurement campaign of $18855 \mathrm{~s}$ real time using a standard 2.2 GHz CPU with 8 GB RAM by use of Matlab Version 2019a. Thus, the ratio of real time to simulation time is determined by 136 . 


\section{Conclusions and outlook}

A real time executable heat transfer model of a solar hydrogen reactor has been developed. The local temperature distribution is calculated in a first model approach with 7 segments for the absorber surface and 1 segment for the quartz window. The dynamic model behavior has been validated by comparing simulation data and measurement results. Several optical parameters like optical view factors were adjusted by a parameter matching routine. The influence of selected sensitive parameters is illustrated by means of model response with different parameter settings. With the presented model version, the temperature distribution is calculated with sufficient accuracy in order to develop control algorithms for automated plant operation.

In ongoing work, the presented model will be linked to additional components to ensure a more detailed model for several purposes. With the resulting overall model, further validation will be carried out by future measurement campaigns and eventual model adaptions in order to improve accuracy. Several model properties are potentially related to tolerances that should be determined by parameter uncertainty studies and additional parameter matching. For example, optical view factors and mean values of the absorber structure like pore size, cellularity and porosity have to be adjusted. Also, experimental fitting parameters of the used Nusselt-Reynolds correlation and reaction rate calculation carried out by use of comparable material must be investigated.

Afterwards, the overall model can be applied for several additional purposes. One aspect is the investigation of the influence of several reactive materials and absorber material properties in relation to an optimal control strategy. Further, mesh refinement studies and convergence checks are carried out to develop high-resolution cell classifications with improved accuracy. Thereby, the local temperature distribution can be calculated in more detail which might be used for optimizing control strategy of heliostat field considering the local temperature distribution and an improvement of absorber design.

Acknowledgements: This work was supported by the LeitmarktAgentur.NRW within the EFRE-Project ASTOR KHH-1-001D.

\section{References}

1. O. Behar, A. Khellaf, K. Mohammedi: A review of studies on central receiver solar thermal power plants, Renewable and
Sustainable Energy Reviews, Vol. 23, P. 12-39, 2013.

2. F. Dähler, M. Wild, R. Schäppi, P. Hauetera, T. Cooper, P. Good, C. Larrea, M. Schmitz, P. Furler, A. Steinfeld: Optical design and experimental characterization of a solar concentrating dish system for fuel production via thermochemical redox cycles, Solar Energy, Vol. 170, P. 568-575, 2018.

3. M. Lin, S. Haussener: Solar fuel processing efficiency for ceria redox cycling using alternative oxygen partial pressure reduction methods, Energy, Vol. 88, P. 667-679, 2015.

4. P. Furler, A. Steinfeld: Heat transfer and fluid flow analysis of a $4 \mathrm{~kW}$ solar thermochemical reactor for ceria redox cycling, Chemical Engineering Science, Vol. 137, P. 373-383, 2015.

5. P. Parthasarathy, P. Le Clercq: Heat transfer simulation in a high temperature solar reactor, Energy Procedia, Vol. 69, P. 1810-1818, 2015.

6. W. Lipinski, J. H. Davidson, S. Haussener, J. F. Klausner, A. M. Mehdizadeh, J. Petrasch, A. Steinfeld, L. Venstrom: Review of heat transfer research for solar thermochemical applications, Journal of Thermal Science and Engineering Applications, Vol. 5, Issue 2, 021005, 2013. https://doi.org/10.1115/1.4024088.

7. J. R. Scheffe, A. Steinfeld: Oxygen exchange materials for solar thermochemical splitting of $\mathrm{H}_{2} \mathrm{O}$ and $\mathrm{CO}_{2}$ : a review, Materials Today, Vol. 17, Issue 7, P. 341-348, 2014.

8. P. Furler, J. Scheffe, D. Marxer, M. Gorbar, A. Bonk, U. Vogt, A. Steinfeld: Thermochemical CO2 splitting via redox cycling of ceria reticulated foam structures with dual scale porosities, Phys. Chem. Chem. Phys., Vol. 16, P. 10503-10511, 2014.

9. C. P. Falter, R. Pitz-Paal: A generic solar-thermochemical reactor model with internal heat diffusion for counter-flow solid heat exchange, Solar Energy, Vol. 144, P. 569-679, 2017.

10. C. Agrafiotis, M. Roeb, C. Sattler: A review on solar thermal syngas production via redox pair-based water/carbon dioxide splitting thermo chemical cycles, Renewable and Sustainable Energy Reviews, Vol. 42, P. 254-285, 2015.

11. J.-P. Säck, S. Breuer, P. Cotelli, A. Houaijia, M. Lange, M. Wullenkord, C. Spenke, M. Roeb, C. Sattler: High temperature hydrogen production: Design of a $750 \mathrm{KW}$ demonstration plant for a two step thermochemical cycle, Solar Energy, Vol.135, P. 232-241, 2016.

12. S. Lorentzou, A. Zygogianni, C. Pagkoura, G. Karagiannakis, A. G. Konstandopoulos et al.: HYDROSOL-PLANT: structured redox reactors for $\mathrm{H}_{2}$ production from solar thermochemical $\mathrm{H}_{2} \mathrm{O}$ splitting, AIP Conference Proceedings, Vol. 2033, Issue 1, 2018.

13. M. Moser, M. Pecchi, T. Fend: Techno-economic assessment of solar hydrogen production by means of thermo-chemical cycles, Energies, Vol. 12, Issue 3, 2019.

14. I. Ermanoski, N. Siegel: Annual average efficiency of a solar-thermochemical reactor, Energy Procedia, Vol. 49, P. 1932-1939, 2014.

15. A. Houaijia, C. Sattler, M. Roeb, M. Lange, S. Breuer, J.-P. Säck: Analysis and improvement of a high-efficiency solar cavity reactor design for a two-step thermochemical cycle for solar hydrogen production from water, Solar Energy, Vol. 97, P. 26-38, 2013.

16. S. Suter, A. Steinfeld, S. Haussener: Pore-level engineering of macroporous media for increased performance of solar-driven thermochemical fuel processing, International Journal of Heat and Mass Transfer, Vol. 78, P. 688-698, 2014.

17. M. Roeb, J.-P. Säck, P. Rietbrock, C. Prahl, H. Schreiber, M. 
Neises, L. de Oliveira et al.: Test operation of a $100 \mathrm{~kW}$ pilot plant for solar hydrogen production from water on a solar tower, Solar Energy, Vol. 85, P. 634-644, 2011.

18. R. J. Panlener, R. N. Blumenthal, J. E. Garnier: A thermodynamic study of nonstoichiometric cerium dioxide, Journal of Physics and Chemistry of Solids, Vol. 36, Issue 11, P. 1213-1222, 1975.

19. Mathworks: https://de.mathworks.com/products/simscape. html, [Online], Day of call: 06.12.2013.

20. D. Laaber, H. v. Storch, K. Wieghardt, H. Fock, C. Sattler, R. Pitz-Paal: One year with Synlight - review of operating experience, AIP Conference Proceedings Vol. 2126, 170007, 2019.

21. Heraeus: http://www.heraeus.com, Manufacturers technical documentation: Quartz Glass for Optics, Optical Properties, [Online], Day of call: 06.12.2019.

22. S. Decker, F. Durst, D. Trimis, S. Nemoda, V. Stamatov, M. Steven, M. Becker, T. Fend, B. Hoffschmidt, O. Reutter: Thermisch beaufschlagte Porenkörper und deren Durchströmungs- und Wärmeübertragungseigenschaften, 2002.

23. B. Bulfin, A. J. Lowe, K. A. Keogh, B. E. Murphy, O. Lübben, S. A. Krasnikov, I. V. Shvets: Analytical model of $\mathrm{CeO}_{2}$ oxidation and reduction, Journal of Physical Chemistry, Vol. 117, Issue 46, P. 24129-24137, 2013.

24. B. Bulfin, F. Call, M. Lange, O. Lübben, C. Sattler, R. Pitz-Paal, I. V. Shvets: Thermodynamics of $\mathrm{CeO}_{2}$ thermochemical fuel production, Energy \& Fuels, Vol. 29, P. 1001-1009, 2015.

25. R. Palumbo, M. Keunecke, S. Möller, A. Steinfeld: Reflections on the design of solar thermal chemical reactors: thoughts in transformation, Energy, Vol. 29, P. 727-744, 2004.

26. R. Müller, A. Steinfeld: Band-approximated radiative heat transfer analysis of a solar chemical reactor for the thermal dissociation of zinc oxide, Solar Energy, Vol. 81, P. 1285-1294, 2007.

27. A. Steinfeld: Radiative transfer in a diffusely/specularly reflecting spherical cavity, Wärme- und Stoffübertragung, Vol. 28, P. 65-68, 1993.

\section{Bionotes}

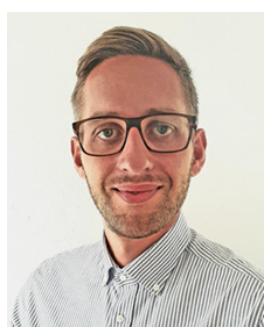

\section{Steffen Menz}

Laboratory of Electrical Power Engineering, Rheinische Fachhochschule Köln (RFH), University of Applied Science, Cologne, Germany

Steffen.Menz@rfh-koeln.de

Steffen Menz is scientific assistant at the University of Applied Sciences RFH in Cologne. His fields of interests are renewable energies, electrical grids, energy management, modeling and simulation of complex systems and hardware development.

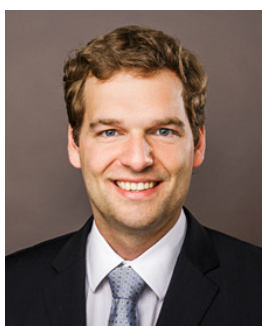

\section{Jörg Lampe}

System theory and Mathematics, Engineering Faculty, Rheinische Fachhochschule Köln (RFH), University of Applied Sciences, Cologne, Germany Joerg.Lampe@rfh-koeln.de

Prof. Jörg Lampe is teaching at the University of Applied Sciences $\mathrm{RFH}$ in Cologne in the areas of simulation, mathematics and system theory. His research interests are modeling and simulation of complex dynamic systems with regards to efficiency and energy flow, as well as availability and reliability in failure analysis.

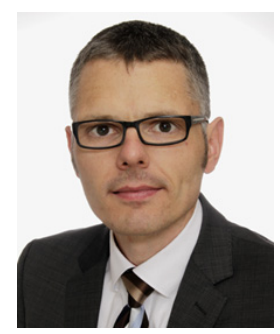

Uwe Tröltzsch

ITK engineering, Cologne, Germany Uwe.Troeltzsch@itk-engineering.de

Dr. Uwe Tröltzsch is expert engineer at ITK engineering. He was teaching electrical and electronic engineering at the University of Applied Sciences RFH in Cologne. His fields of interest are modeling and simulation of technical and natural systems. These aspects he applies in the development of embedded systems including electronics and software.

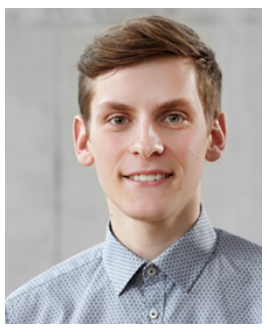

\section{Philipp Weiler}

Mechanical Engineering, Rheinische Fachhochschule Köln (RFH), University of Applied Science, Cologne, Germany philipp_johannes_weiler@smail.thkoeln.de

Philipp Weiler has studied Mechanical Engineering at the University of Applied Sciences RFH in Cologne in the bachelor program. He worked as a research assistant in the research project ASTOR before starting his master studies in renewable energy at the Technical University of Cologne. His research interests are the physical simulation of regenerative energy systems with a focus on radiation physics. 


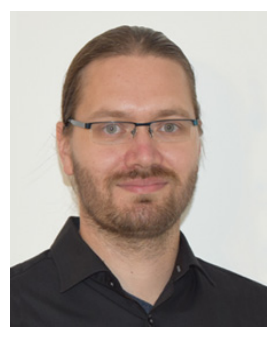

\section{Arne Pahl}

Fundamentals of Electrical Engineering, Helmut Schmidt University Hamburg, Hamburg, Germany Arne.Pahl@hsu-hh.de

Arne Pahl worked until September 2019 at the University of Applied Science RFH in Cologne in the laboratory for electrical energy technology. Since then he has been working at the Institute for Fundamentals of Electrical Engineering at Helmut Schmidt University Hamburg in the research field of Electromagnetic Compatibility.

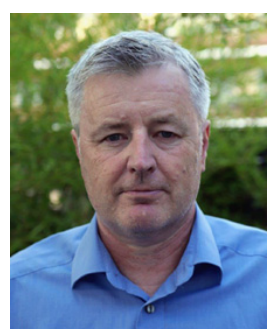

\section{Thomas Fend}

German Aerospace Center (DLR), Institute of Solar Research, Cologne, Germany

Thomas.Fend@dlr.de

Thomas Fend is Project Manager in the department of Solar Process Engineering and is involved in the development of thermochemical technologies for hydrogen production, receivers for solar thermal power plants and new methods for $\mathrm{CO} 2$ capture.

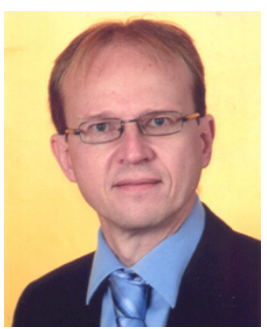

Thomas Seeger

University of Siegen, Engineering

Thermodynamics, D-57076 Siegen, Germany

Center for Sensor Systems (ZESS),

University of Siegen, 57076 Siegen,

Germany

Thomas.Seeger@uni-siegen.de

Since 2010 Prof. Dr.-Ing. Thomas Seeger is full Professor for Engineering Thermodynamics at the Faculty IV (Science and Technology), University of Siegen. 